\title{
Biomarkers role in prognosis and survival outcomes in patients with renal tumors: review article
}

Volume 4 Issue $6-2017$

Keywords: renal cell carcinoma, immunohistochemical, biomarkers, metastatic

\section{Introduction}

Renal cell carcinoma (RCC) is one of the most common urological malignancies. In 2016, approximately 62,700 adults (39,650 males and 23,050 females) were diagnosed with RCC in the US and approximately 14,240 individuals died from renal neoplasm. ${ }^{1}$ RCC mortality rate can vary between $26 \%$ up to $94 \%$ according to stage and aggressiveness of the disease. ${ }^{2} \mathrm{RCC}$ accounts for approximately $3 \%$ of solid tumors with high mortality rate due to the absence of curative therapy for advanced disease. ${ }^{3} \mathrm{RCC}$ can be classified as nonepithelial or epithelial, according to cell origin. The four major types are of epithelial origin includes: clear cell renal carcinoma (ccRCC), papillary, chromophobe renal carcinoma (chRCC) and collecting duct carcinoma. The most common subtype of RCC is ccRCC which accounts for approximately $70-80 \%$ of all RCC tumors. ${ }^{4,5}$ Chromophobe renal cell carcinoma is accounts for approximately $3-5 \%$ with 5 -year survival rate up to $94 \%{ }^{6}$ The standard treatment of localized ccRCC patients is either partial or radical nephrectomy, however, metastasis has been shown to develop in approximately $30 \%$ of patients after surgery. ${ }^{7,8}$ Metastatic ccRCC may require immunotherapy, targeted therapy, and/or radiation therapy but with low success rate which typically leaves no other option except for surgery. ${ }^{\text {? }}$

Considering metastatic ccRCC has a low 5-year disease free survival rate $(<10 \%)$, early diagnosis and prognostic data is critical in order to achieve the full benefit of surgery and increase the survival outcomes. ${ }^{79}$ Prognosis for ccRCC can be determined by many factors including; cancer grade, stage, response and resistance to treatment. With current imaging modalities, renal tumors including ccRCC can be easily identified at early stage; however, these imaging modalities cannot identify the tumor stage?

The potential for utilizing immunohistochemistry in RCC subtypes classification has shown valuable diagnostic and prognostic significance. ${ }^{10}$ However, currently there is no consensus in regard to what biomarker should be used in RCC classification. ${ }^{10}$ Various RCC associated biomarkers have been studied including PAX-2/PAX8, CAIX, adipophillin (ADP), CD10, and monoclonal antibody RCC marker (RCCma). ${ }^{11}$ PAX, CAIX and vimentin are biomarkers that might be promising in RCC prognostic significance nowadays.

In particular, PAX-2 and PAX-8 have high sensitivity and specificity for RCC tumors. PAX-2 and PAX- 8 are members of paired box gene family which consists of nine members where each one encodes a transcription factor that is expressed during fetal development. ${ }^{12-14}$ These transcription factors play an important role in the formation of organs and tissues during embryonic development and subsequent maintenance of certain cells after birth. ${ }^{12,13}$ PAX8 oversees renal and thyroid organogenesis. ${ }^{14}$ Specifically, PAX-2 and PAX- 8 are generously expressed by renal blastemal cells during

\author{
Mahmoud Mohamed \\ New York Presbyterian Brooklyn Methodist Hospital, USA
}

Correspondence: Mahmoud Mohamed, New York Presbyterian Brooklyn Methodist Hospital New York, NY I I I 5 , USA, Tel I 718780 5616, Email ma.elhagagy@gmail.com

Received: May 22, 2017 | Published: June 02, 2017

nephrogenesis. In adulthood, this expression mostly disappears or is present only in few parenchymal cells; however, expression is increased in RCC. ${ }^{12,13}$ Recent studies evaluated the sensitivity and specificity for each marker has a diagnostic value for ccRCC. The study contained 26 cases of ccRCC and 51 cases of non-renal cancer with clear cell morphology. PAX-2 was positive to ccRCC by $81 \%$ and was positive in $24 \%$ of clear cell morphology group. PAX- 8 in ccRCCgroup was $100 \%$ positive and was $39 \%$ positive in the clear cell morphology group. ${ }^{11}$ The study concluded that PAX-2 and PAX-8 are sensitive markers to ccRCC and they lose their specificity in the presence of mullerian tumor cells. ${ }^{11}$

On the other hand, carbonic anhydrase IX (CAIX) is released under hypoxic conditions caused by the upstream hydroxylation of hypoxiainducible factor 1-alpha (HIF-1alpha) and subsequent binding to the Von Hippel Lindau (VHL) protein. ${ }^{15,16}$ Over-expression of CAIX is therefore associated with various tumor malignancies including ccRCC. ${ }^{17,18}$ CAIX is expressed in both high grade and low grade RCC. The CAIX immunohistochemical membrane staining pattern for ccRCC is circumferential while pRCC typically lacks tumor cell apical surface staining and is positive for basolateral membrane outlining. ${ }^{19,20}$ Low grade ccRCC immunohistochemical testing can include KIM-1, vimentin, and CAIX. Low grade renal neoplasms that exhibit both clear cell/granular features typically have a negative CAIX expression result which can be diagnostic for chromophone renal cell carcinoma (ChRCC) and oncocytoma. ${ }^{20}$ Given its diagnostic association with RCC, the prognostic value of CAIX has received attention. Specifically, several studies have suggested that high CAIX expression $(\geq 75 \%)$ may be able to predict prognostic outcomes in patients with ccRCC. ${ }^{16,21,22,23}$ In addition, high CAIX expression has also been cited as an independent predictor for improved interleukin-2 therapy response. ${ }^{24}$ Conversely, low CAIX expression ( $\left.<75 \%\right)$ was associated with higher mortality and a greater chance of lymphatic spread. ${ }^{15,16}$

By examining tumor pathologic features, some studies have found inconsistent results for CAIX expression and patient survival..$^{16,24,25}$ Survival outcomes in patients with RCC can be affected by patient 
background, clinical symptoms, and tumor pathologies such as tumor necrosis and nuclear grade. ${ }^{16,26}$ Variations in the findings of many reported studies in results continue to generate controversy over CAIX expression and patient prognosis significance. ${ }^{16}$ Finally, vimentin, which is a type III intermediate filament that is expressed in normal mesenchymal cells and helps provide resistance to stress. ${ }^{27}$ In addition to renal tubular and renal stromal cells, Vimentin expression is recognized in broad scope of other cell types including Sertoli cell, trophoblasts, giant cells, pancreatic cells, endothelial cells, fibroblast, macrophages, neutrophils, leukocytes and mesangial cells. ${ }^{28,29}$ Overexpression of vimentin has been detected in prostate cancer, lung cancer, gastrointestinal cancer, and malignant melanoma. Although vimentin has been used for diagnosing and classifying renal neoplasms, there is currently no consensus regarding which immunomarker can be used as standards for renal neoplasm classification. Moreover, little is known regarding vimentin's prognostic value in renal tumors. Considering the identification of specific renal tumor type is crucial in determining treatment and prognosis, biomarkers capable of differential diagnosis and prognostic valueare of great interest.

Vimentin is reported to be an essential marker for epithelialmesenchymal transition (EMT). EMT is associated with numerous tumorigenic incidents, which vimentin overexpression occurs. However, vimentin exact role remains unknown. ${ }^{4,30}$ EMT is also related to tumor invasion, proliferation and metastasis. ${ }^{31}$ Over expression of vimentin is associated with epithelial cancers including ccRCC and correlates with tumor growth, invasion, and poor prognosis; however, its role in cancer progression remains uncertain. ${ }^{27}$ Vimentin is considered as a potential molecular target for cancer therapy but its specific role remains unclear as well., ${ }^{4,27}$ Moreover, vimentin is used as a reliable biomarker in differentiating certain types of renal cell carcinomas..$^{32}$ It has been shown that vimentin is expressed in the majority of clear cell and papillary renal cell carcinomas; however, it is rarely expressed in oncocytoma and chromophobe renal cell carcinomas. ${ }^{32,33}$ Therefore, vimentin may assist in confirming diagnoses and having a prognostic value regarding each subtype of RCC. Vimentin overexpression was specific to ccRCC in comparison to other chRCC and oncocytoma. A study presented at the American Association of Cancer research (AACR) annual conference in 2016included 66 patients with ccRCC, 11 patients with Oncocytoma and 11 patients with chRCC reported that Vimentin overexpression may be a marker of metastatic disease in patients with ccRCC. ${ }^{34}$ Vimentin expression was not significant for tumor grade, pathologic stage, or presence of sarcomatoid features in ccRCC. ${ }^{34}$

\section{Conclusion}

The efficiency and accuracy of biomarkers studies using immunohistochemical and tissue microarray techniques are still variable and unclear in regards to prognostic significance and survival outcomes in patient with renal tumors. Multiple biomarkers shown to be significant to assess diagnosis and prognosis in these patients and other were not significant. Studies with larger sample sizes and more specific to each biomarker will be needed to validate the results and confirm the usefulness of biomarkers in regards to prognostic significance in patients with renal tumors.

\section{References}

1. American Cancer Society. Cancer Facts and Figures 2016. Atlanta, Ga: American Cancer Society, USA. 2016.

2. Motzer RJ, Hutson TE, Tomczak P, et al. Overall survival and updated results for sunitinib compared with interferon alfa in patients with metastatic renal cell carcinoma. J Clin Oncol. 2009;27(22):3584-3590.
3. Jemal A, Siegel R, Ward E, et al. Cancer statistics. CA Cancer J Clin. 2007;57(1):43-66.

4. Shi ZG, Li SQ, Li ZJ, et al. Expression of Vimentin and surviving in clear cell renal cell carcinoma and correlation with p53. Clin Transl Oncol. 2015;17(1):65.

5. Crotty TB, Farrow GM, Lieber MM. Chromophobe cell renal carcinoma: clinicopathological features of 50 cases. J Urol. 1995;154(3):964-967.

6. Thoenes W, Storkel S, Rumpelt HJ, et al. Chromophobe cell renal carcinoma and its variants-a report on 32 cases. J Pathol. 1988;155(4):277-287.

7. Cooper SJ, Tun HW, Roper SM, et al. Current status of biomarker discovery in human clear cell renal cell carcinoma. J Mol Biomark Diagn. 2012;S1:2.

8. Pantuck AJ, Zisman A, Belldegrun AS. The changing natural history of renal cell carcinoma. J Urol. 2001;166(5):1611-1623.

9. Weiss RH, Lin PY. Kidney cancer: identification of novel targets for therapy. Kidney Int. 2006;69(2):224-232.

10. Tan PH, Cheng L, Rioux-Leclercq N, et al. Renal tumors: diagnostic and prognostic biomarkers. Am J SurgPathol. 2013;37(10):1518-1531.

11. Mentrikoski MJ, Wendroth SM, Wick M. Immunohistochemical Distinction of Renal Cell Carcinoma from Other Carcinomas with Clear-Cell Histomorphology: Utility of CD10 and CA-125 in Addition to PAX-2, PAX-8, RCCma, and Adipophilin. Appl Immunohistochem Mol Morphol. 2014;22(9):635-641.

12. Mansouri A, Stoykova A, Torres M, et al. Dysgenesis of cephalic neural crest derivatives in Pax7-/- mutant mice. Development. 1996;122(1996):831-838.

13. Dahl E, Koseki H, Balling R. Pax genes and organogenesis. BioEssays. 1997;19(1997):755-765.

14. Barr ML, Jilaveanu LB, Camp RL, et al. Pax-8 expression in renal tumors and distant sites: A useful marker of primary and metastatic renal cell carcinoma? J Clin Pathol. 2015;68(1):12-17.

15. Ivanov S, Liao SY, Ivanova A, et al. Expression of hypoxia-inducible cell-surface transmembrane carbonic anhydrases in human cancer. Am J Pathol. 2001;158(3):905-919.

16. Stillebroer AB, Mulders PF, Boerman OC, et al. Carbonic anhydrase IX in renal cell carcinoma: implications for prognosis, diagnosis, and therapy. Eur Urol. 2010;58(1):75-83.

17. Swinson DE, Jones JL, Richardson D, et al. Carbonic anhydrase IX expression, a novel surrogate marker of tumor hypoxia, is associated with a poor prognosis in non-small-cell lung cancer. J Clin Oncol. 2003;21(3):473-482.

18. Genega EM, Ghebremichael M, Najarian R, et al. Carbonic anhydrase IX expression in renal neoplasms: correlation with tumor type and grade. Am J Clin Pathol. 2010;134(6):873-879.

19. Tan PH, Cheng L, Rioux-Leclercq N, et al. Renal tumors: diagnostic and prognostic biomarkers. Am J Surg Pathol. 2013;37(10):1518-1531.

20. Wilkerson ML, Lin F, Liu $H$, et al. The application of immunohistochemical biomarkers in urologic surgical pathology. Arch Pathol Lab Med. 2014;138(12):1643-1665.

21. Bui MH, Seligson D, Han KR, et al. Carbonic anhydrase IX is an independent predictor of survival in advanced renal clear cell carcinoma: implications for prognosis and therapy. Clin Cancer Res. 2013;9(2):802-811.

22. Sandlund J, Oosterwijk E, Grankvist K, et al. Prognostic impact of carbonic anhydrase IX expression in human renal cell carcinoma. BJU Int. 2007;100(3):556-560. 
23. Patard JJ, Fergelot P, Karakiewicz PI, et al. Low CAIX expression and absence of VHL gene mutation are associated with tumor aggressiveness and poor survival of clear cell renal cell carcinoma. Int $J$ Cancer. 2008;123(2):395-400.

24. Atkins M, Regan M, McDermott D, et al. Carbonic anhydrase IX expression predicts outcome of interleukin 2 therapy for renal cancer. Clin Cancer Res. 2005;11(10):3714-3721.

25. Chamie K, Klopfer P, Bevan P, et al. Carbonic anhydrase-IX score is a novel biomarker that predicts recurrence and survival for high-risk, nonmetastatic renal cell carcinoma: data from the phase III ARISER clinical trial. Urol Oncol. 2015;33(5):204.e25-e33.

26. Galfano A, Novara G, Iafrate M, et al. Mathematical models for prognostic prediction in patients with renal cell carcinoma. Urol Int. 2008;80(2):113-123.

27. Hes O, Michal M, Kuroda N, et al. Vimentin reactivity in renal oncocytoma: immunohistochemical study of 234 cases. Arch Pathol Lab Med. 2007;131(12):1782-1788.

28. Carter V, Shenton BK, Jaques B, et al. Vimentin antibodies: a non-HLA antibody as a potential risk factor in renal transplantation. Transplant Proc. 2005;37(32):654-657.
29. Ko SH, Suh SH, Kim BJ, et al. Expression of the intermediate filament vimentin in proliferating duct cells as a marker of pancreatic precursor cells. Pancreas. 2004;28(2):121-128.

30. Satelli A, Li S. Vimentin in cancer and its potential as a molecular target for cancer therapy. Cell Mol Life Sci. 2011;68(18):3033-3046.

31. Shirahata A, Sakata M, Sakuraba K, et al. Vimentin methylation as a marker for advanced colorectal carcinoma. Anticancer Res. 2009;29(1):279-281.

32. Palsdottir HB, Hardarson S, Petursdottir V, et al. Incidental detection of renal cell carcinoma is an independent prognostic marker: results of a long-term, whole population study. J Urol. 2014;187(1):48-53.

33. Wilkerson ML, Lin F, Liu H, et al. The Application of Immunohistochemical Biomarkers in Urologic Surgical Pathology. Arch Pathol Lab Med. 2014;138(12):1643-1665.

34. Mahmoud Mohamed, Lulin Hu, Haiyan Liu, et al. Vimentin expression as a potential immunomarker of predicting aggressive disease in clear cell renal cell carcinoma. Proceedings of the American Association for Cancer Research. 2016;57(1):392. 